
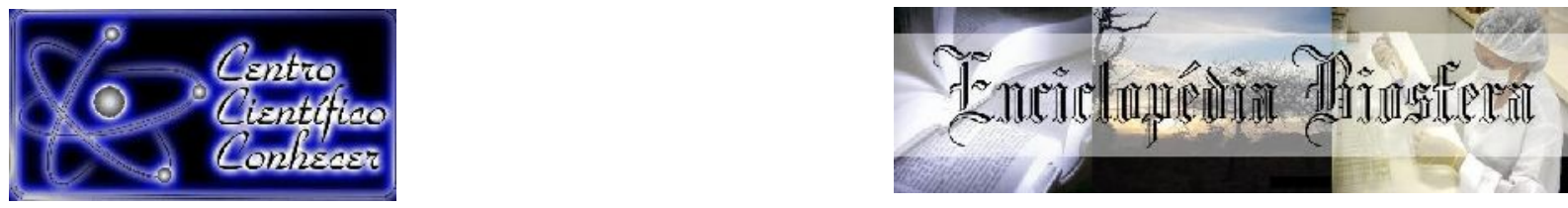

\title{
ANÁLISE DO CARBONO ESTOCADO DE INDIVÍDUOS PERFILHADOS CONSIDERANDO DIFERENTES METODOLOGIAS
}

\author{
Rafaella Tavares Pereira ${ }^{1}$, Natielle Gomes Cordeiro ${ }^{2}$, Marcela de Castro Nunes Santos \\ Terra $^{3}$, José Márcio de Mello ${ }^{4}$ \\ ${ }^{1}$ Graduanda em Engenharia Florestal pela Universidade Federal de Lavras - UFLA, \\ Lavras, Minas Gerais, Brasil. \\ ${ }^{2}$ Doutoranda em Engenharia Florestal pela Universidade Federal de Lavras - UFLA, \\ Lavras, Minas Gerais, Brasil. E-mail: natiellegcordeiro@gmail.com \\ ${ }^{3}$ Doutora em Engenharia Florestal pela Universidade Federal de Lavras - UFLA, \\ Lavras, Minas Gerais, Brasil. \\ ${ }^{4}$ Professor Titular pela Universidade Federal de Lavras - UFLA Lavras, Minas Gerais, \\ Brasil.
}

\section{Recebido em: 15/08/2020 - Aprovado em: 15/09/2020 - Publicado em: 30/09/2020 DOI: 10.18677/EnciBio_2020C5}

\begin{abstract}
RESUMO
As florestas, além da rica biodiversidade, estocam o carbono da atmosfera na biomassa acima e abaixo do solo. A biomassa acima do solo (AGB) é fundamental para a quantificação do carbono. Deste modo, o objetivo desse trabalho foi analisar o estoque de carbono entre os anos de 2015 e 2017, bem como a contribuição de cada espécie e comparar a variação na característica de interesse por meio de duas metodologias diferentes quanto ao perfilhamento dos indivíduos. A área de estudo localiza-se em um remanescente florestal no município de Lavras, Minas Gerais. Foram consideradas duas metodologias para a quantificação do estoque de carbono, em que a primeira considera cada perfilho como um indivíduo e a segunda em que se considera a transformação dos diâmetros em diâmetro geométrico. Estimou-se a AGB e o estoque de carbono para o fragmento. Por fim, analisou-se o Incremento Periódico Anual de Carbono. No total, houve um decréscimo de $1,34 \%$ da quantidade de carbono nos dois anos de estudo. Observou-se que o método considerando cada bifurcação como uma árvore obteve uma diferença de $1,25 \%$ a mais quando comparada com a forma comum de se tratar uma árvore bifurcada em análises da vegetação nativa. As espécies Copaifera langsdorffii Desf. e Xylopia brasiliensis Spreng. corresponderam em média $18,57 \%$ e $13,63 \%$ da porcentagem total de carbono, representando grande importância ao fragmento.
\end{abstract}

PALAVRAS-CHAVE: Assimilação de carbono; Biomassa acima do solo; Gases de efeito estufa. 


\title{
ANALYSIS OF CARBON STORED IN TREE TILLER INDIVIDUALS CONSIDERING DIFFERENT METHODOLOGIES
}

\begin{abstract}
The forests, in addition to rich biodiversity, store carbon from the atmosphere in biomass above and below ground. The above-ground biomass (AGB) is essential for the quantification of carbon. Thus, this study aimed to analyze the carbon stock between the years of 2015 and 2017, as well as the contribution of each species and to compare the variation in the variable of interest by means of two different methodologies regarding the tillering of individuals. The study area is located in a forest remnant in the municipality of Lavras, Minas Gerais state. It was considered two methodologies for the carbon stock quantification, in which the first considers each tiller as an individual and the second in which it was considered the transformation of diameters into geometric diameter. The AGB and carbon stock for the fragment were estimated. Finally, it analyzed the Annual Periodic Carbon Increase. In total, there was a $1.34 \%$ decrease in the amount of carbon in the two years of study. It was observed that the method considering each bifurcation as a tree obtained a difference of $1.25 \%$ more when compared to the common way of treating a bifurcated tree in analyzes of native vegetation. The species Copaifera langsdorffii Desf. and Xylopia brasiliensis Spreng. corresponded, on average, $18.57 \%$ and $13.63 \%$ of the total carbon percentage, representing great importance to the fragment.
\end{abstract}

KEYWORDS: Carbon assimilation; Above-ground biomass; Greenhouse gases

\section{INTRODUÇÃO}

A realização de atividades antrópicas de forma desenfreada tem ocasionado consequências negativas aos ecossistemas florestais (SILVEIRA et al., 2019a). Dentre as atividades mais desenvolvidas, tem-se o desmatamento para terras agricultáveis e a queima de combustíveis fósseis para a indústria, causando aumento da temperatura da Terra, e mudanças climáticas globais (VITAL, 2018). Tais fatores despertam uma crescente preocupação em relação às emissões de $\mathrm{CO}_{2}$ na atmosfera, fomentando assim diversos acordos internacionais na redução dos níveis de emissões de carbono pelos países no mundo (SOUZA; CORAZZA, 2017; VITAL, 2018).

Entre os acordos internacionais visando o combate às extremas emissões de carbono, há o Protocolo de Quioto de 1992 e o Acordo de Paris em 2005 (VITAL, 2018). No Protocolo de Quioto, apenas os países desenvolvidos participaram do acordo para reduzir as emissões, sem a participação dos demais (EIBEL; PINHEIRO, 2016). Em relação ao Acordo de Paris, este não foi eficaz devido à ausência de punições para os países que não obedeceram aos princípios estabelecidos (VITAL, 2018). Contudo, a grande vantagem dos acordos foi a conscientização em relação ao meio ambiente e a possibilidade de financiamentos para ações de combate às mudanças climáticas de países desenvolvidos em países em desenvolvimento (ONU, 2015).

Os acordos supracitados se fizeram de suma importância para as negociações de crédito de carbono no Brasil, por meio do Mecanismo de Desenvolvimento Limpo (MDL), em que as Unidades de Redução de Emissões são vendidas para os países desenvolvidos que não cumpriram suas metas de redução de carbono. Considerando que o Brasil é isento, além de obter as Unidades de Redução de Emissões para vendê- 
las, há um fluxo financeiro no País, a criação de projetos sustentáveis, bem como a provisão de serviços ecossistêmicos (GROOT et al., 2010). De acordo com Kumar e Mutanga (2017), a biomassa florestal começou a ter maior importância nos dias atuais devido as negociações de crédito de carbono. Assim, as Unidades de Redução de Carbono se tornam uma ferramenta para que a sustentabilidade e o desenvolvimento econômico caminhem juntos (EIBEL; PINHEIRO, 2016).

Os passivos ambientais e a compensação ambiental também foram auxiliares nas questões sustentáveis do Brasil. Nesse sentido, a criação de unidades de conservação devido aos impactos antropológicos com perdas de espécies vegetais e animais consolidou o Sistema Nacional de Unidades de Conservação da Natureza (SNUC) (FARIA, 2008). Também, medidas como o Cadastro Ambiental Rural (CAR) (OLIVEIRA; FERNANDES-FILHO, 2016), criação de Reservas Legais (RL), Áreas de Preservação Permanente (APP) e a criação do Pagamento por Serviço Ambiental (PRA) são importantes processos de restauração, conservação e manejo sustentável, que auxiliam nas reduções de gases de efeito estufa.

Sendo assim, as formações naturais desempenham um papel importante no armazenamento de carbono e conservação da biodiversidade (SULLIVAN et al., 2017; CORDEIRO et al., 2018, 2019, 2020; SILVEIRA et al., 2019b). O armazenamento de carbono na biomassa das florestas, composta por material vivo e morto, é realizado tanto na biomassa acima como abaixo do solo (ZELARAYÁN et al., 2015). Destaca-se que, embora não seja exclusivamente de material vivo, a biomassa acima do solo (no inglês above-ground biomass - AGB) é um intermediário importante na quantificação do carbono em florestas (KENZO et al., 2015; ADAIR et al., 2018), já que as folhas, galhos e sementes acima do solo estão em maior proporção em relação à biomassa abaixo do solo (KUMAR; MUTANGA, 2017), que por sua vez é de mais difícil quantificação (SANFORD; CUEVAS, 1996).

Quantificar o estoque de carbono das formações vegetais é possível a partir do método destrutivo, delimitado pelas características físicas e ambientais da área como tamanho e vegetação, ou com o método baseado em estimativas de equações alométricas que empreende o uso das características mensuradas no campo, (NAM et al., 2016; KUMAR; MUTANGA, 2017; MIRANDA et al., 2019). Por exemplo, cita-se o cálculo AGB por meio de equações alométricas fornecidas por Chave et al. (2014), que vem sendo amplamente utilizada (JOHNSON et al., 2016; TANAGO et al., 2018; CORDEIRO et al., 2019). Porém, ainda há poucas equações de estimativas confiáveis de quantificação da biomassa (OLIVEIRA et al., 2016), mesmo este parâmetro sendo bastante atrativo devido a sua abundância e utilização (KUMAR; MUTANGA, 2017).

Um dos pontos chave da utilização de equações alométricas para o cálculo da biomassa é a organização da base de dados. Nesse sentido, há duas possibilidades na consideração de indivíduos bifurcados ou perfilhados: considerar os fustes individualmente ou fazer o cálculo do chamado "diâmetro geométrico" dos fustes, que consiste na raiz da soma dos quadrados dos diâmetros dos fustes (MIRANDA et al., 2011). O impacto das diferentes abordagens na quantificação do carbono em florestas foi pouco explorado até então. Desse modo, o presente estudo teve como objetivos: i) analisar a quantidade de carbono no remanescente florestal entre os anos de 2015 e 2017; ii) analisar a contribuição de carbono das espécies no remanescente; iii) 
comparar a variação do estoque de carbono entre indivíduos perfilhados considerando o cálculo do diâmetro geométrico e considerando-os como fustes individuais.

\section{MATERIAL E MÉTODOS}

A área de estudo localiza-se em um remanescente florestal no município de Lavras, na região sul de Minas Gerais, na Universidade Federal de Lavras. O remanescente possui 6,3 hectares e está localizado entre as coordenadas 2113'40"S e 4457'50'W. A fitofisionomia é classificada em Floresta Estacional Semidecidual Montana (FESM), com presença de bromélias, cipós e trepadeiras.

O fragmento possui solo do tipo latossolo vermelho distrófico com textura fina, relevo levemente ondulado e altitude de $925 \mathrm{~m}$. O clima é classificado como Cwa de acordo com a classificação climática de Köppen, sendo assim caracterizado com verões quentes e invernos secos, com temperaturas acima de $22^{\circ} \mathrm{C}$ nos verões. A precipitação anual média da região é de 1461,8 mm (ALVARES et al., 2013).

Para a coleta dos dados, foi realizado um inventário no remanescente nos anos de 2015 e 2017. Para isso, procedeu-se com a alocação de 126 parcelas de 20x20 m², onde foram mensurados todos os indivíduos com diâmetro à 1,30 metros do solo (DAP) igual ou maior que $5 \mathrm{~cm}$. No primeiro ano, todos os indivíduos que atenderam o critério de inclusão foram identificados com placas de alumínio contendo o número da planta e da parcela para posterior mensuração. Também, entre os anos de inventário, foi feita a identificação botânica de todos os indivíduos mensurados, além da identificação de recrutas e árvores mortas no remanescente.

Visando a quantificação do estoque de carbono, assim como realizar uma comparação na determinação da variável, foram consideradas duas metodologias. Inicialmente, considerou-se cada árvore bifurcada como um indivíduo e, posteriormente, cada bifurcação foi considerada um indivíduo. Tais procedimentos foram realizados para os dois anos de inventário. Em relação aos indivíduos bifurcados, estes tiveram o diâmetro transformado em um só valor utilizando a seguinte fórmula (MACDICKEN et al., 1991):

$$
D=\sqrt{d_{n 1}^{2}+d_{n 2}^{2}+\cdots+d_{n}^{2}}
$$

Em que: $D=$ Diâmetro geométrico; $d_{n}=$ diâmetro dos perfilho das plantas bifurcadas.

Em relação a quantificação do estoque de carbono, inicialmente estimou-se a biomassa acima do solo (Above-ground biomass - AGB) por meio do software $R$ ( $R$ CORE TEAM, 2020), utilizando o pacote BIOMASS (RÉJOU-MÉCHAIN et al., 2017). Esse pacote é baseado no modelo proposto por Chave et al. (2014) (Equação 1). Ressalta-se que o modelo leva em consideração, como variáveis independentes, o diâmetro (D) e a densidade da madeira (WD), esta obtida no banco de dados The Global Wood Density (GWD) (ZANNE et al., 2009).

$$
A G B=\exp \left(-2.024-0.896 E+0.920^{\star} \log (W D)+2.795^{\star} \log (D)-0.0461\left(\log (D)^{2}\right)\right) \quad(E q .1)
$$

Em que: $E=$ Estresse ambiental gerado pelas coordenadas geográficas; $W D=$ Densidade da madeira; $\mathrm{D}=$ Diâmetro à 1,30 metros do solo (DAP). 
Posteriormente ao cálculo da AGB, o valor de cada indivíduo foi multiplicado por 0,471 no Excel 2013, obtendo assim o estoque de carbono por indivíduo, por parcela e para o fragmento nos dois anos de estudo. Esse fator multiplicativo é o teor de carbono médio nas angiospermas (SULLIVAN et al., 2017).

Ademais, o Incremento Periódico Anual de Carbono foi calculado para os dois métodos de comparação, ou seja, calculando o diâmetro geométrico para árvores bifurcadas e considerando cada bifurcação como uma árvore.

\section{RESULTADOS E DISCUSSÃO}

A partir do inventário florestal realizado, foram mensurados e identificados 5.528 e 5.122 indivíduos para os anos de 2015 e 2017, respectivamente, distribuídos em 185 espécies, 106 gêneros e 48 famílias. O número de árvores mortas no fragmento foi 604 e o número de recrutas 54 .

As famílias que mais contribuíram com o armazenamento de carbono no fragmento, nos dois anos de inventário, foram Fabaceae, Lauraceae, Annonaceae, Melastomataceae e Myrtaceae (Tabela 1). Assim, essas principais famílias representaram 82,14\% e 82,66\% do carbono total em 2015 e 2017, respectivamente.

TABELA 1 - Estimativa do estoque de carbono, em toneladas, para as famílias mais representativas nos anos de 2015 e 2017 em um remanescente de Floresta Estacional Semidecidual Montana em Lavras, Minas Gerais.

\begin{tabular}{ccccc}
\hline Família & $\mathrm{C}(2015)$ & $\%(2015)$ & $\mathrm{C}(2017)$ & $\%(2017)$ \\
\hline Fabaceae & 134,77 & 30,73 & 133,27 & 30,80 \\
Lauraceae & 114,38 & 26,08 & 111,19 & 25,70 \\
\hline Annonaceae & 65,20 & 14,87 & 70,26 & 16,24 \\
\hline Melastomataceae & 29,02 & 6,62 & 26,55 & 6,14 \\
\hline Myrtaceae & 16,88 & 3,85 & 16,36 & 3,78
\end{tabular}

Em que: $C(2015)$ = quantidade de carbono, em toneladas, em 2015; \% (2015) = porcentagem de carbono por família em relação ao total em 2015; C (2017) = quantidade de carbono, em toneladas, em 2017; \% (2017) = porcentagem de carbono por família em relação ao total em 2017.

Em relação as espécies estudadas, realizou-se um ranqueamento das 10 espécies que apresentaram maior contribuição ao estoque de carbono, sendo estas explicitadas na Tabela 2. Entre as espécies, Copaifera langsdorffii Desf. (Fabaceae) e Xylopia brasiliensis Spreng. (Annonaceae) obtiveram destaque, representando respectivamente $18,40 \%$ e 12,96\% em relação ao carbono total em 2015 e 18,72\% e 14,30\% em 2017. Essas espécies representaram 31,36\% em 2015 e 7,63\% em 2017. Além disso, foi observado que essas espécies ranqueadas pertencem as famílias mais representativas no remanescente, com exceção da Myrtaceae. De acordo com Pedroni et al. (2002), ocorre maior ganho em estoque de carbono para a espécie Copaifera langsdorffii Desf. ao se considerar suas características morfológicas e fenotípicas no período chuvoso. 
TABELA 2 - Dez espécies arbóreas ranqueadas pelo estoque de carbono, em toneladas, para os anos de 2015 e 2017 em um remanescente de Floresta Estacional Semidecidual Montana em Lavras, Minas Gerais.

\begin{tabular}{ccccc} 
Espécies & C (2015) & $\%(2015)$ & C (2017) & $\%(2017)$ \\
\hline Copaifera langsdorffii Desf. & 80,72 & 18,40 & 81,01 & 18,72 \\
\hline Xylopia brasiliensis Spreng. & 56,83 & 12,96 & 61,88 & 14,30 \\
\hline Cryptocarya aschersoniana Mez & 36,06 & 8,22 & 38,15 & 8,81 \\
Ocotea corymbosa (Meisn.) Mez & 30,41 & 6,93 & 30,26 & 6,99 \\
\hline Miconia willdenowii Klotzsch ex & 26,91 & 6,13 & 24,45 & 5,65 \\
\hline Naudin & 23,06 & 5,25 & 21,32 & 4,92 \\
\hline Ocotea odorifera (Vell.) Rohwer & 22,21 & 5,06 & 20,24 & 4,67 \\
\hline Tachigali rugosa (Mart. ex Benth.) & Zarucchi \& Pipoly & 4,60 & 16,63 & 3,84 \\
\hline Persea major (Meisn.) L.E.Kopp & 20,18 & 3,12 & 14,34 & 3,31 \\
\hline Maprounea guianensis Aubl. & 13,69 & 3,05 & 13,12 & 3,03 \\
\hline Hymenaea courbaril L. & 13,40 & 3,05 & & \\
\hline
\end{tabular}

No remanescente, foram calculadas as quantidades de carbono (C) nos anos de 2015 e 2017. Em 2015, o estoque de carbono presente foi de 438,65 toneladas, o que representa 69,62 ton.ha ${ }^{-1}$, enquanto em 2017 a quantidade total foi de 432,76 toneladas, o que equivale a 68,69 ton.ha $^{-1}$. Esse decréscimo corresponde $1,34 \%$ da quantidade de carbono perdida nos dois anos de estudo e este resultado pode estar atrelado ao número de árvores mortas entre os anos de mensuração.

A estimativa de quantidade de carbono na FESM está próxima ao estudo de Torres et al. (2013), que encontraram 72,80 ton.ha $^{-1}$ com o uso de equações proposta pelo Painel Intergovernamental sobre Mudanças do Clima (IPPC), que estimaram a biomassa através da AGB e equações alométricas.

Para outros trabalhos em FESM utilizando equações alométricas com diferentes estimativas de biomassa, as estimativas desse estudo se encontram inferiores. Carvalho et al. (2014) encontraram 96,74 ton.ha' ${ }^{-1}$ em Ribeirão Grande, São Paulo e Amaro et al. (2013) encontraram um valor de 86,93 ton.ha ${ }^{-1}$ em Viçosa, Minas Gerais. As estimativas foram superiores em relação ao encontrado por Torres et al. (2017), de 30,99 ton.ha ${ }^{-1}$.

No estudo de Lima et al. (2003), foi encontrado 28,8 ton.ha ${ }^{-1}$ em um fragmento de floresta estacional semidecidual secundária na Fazenda Experimental do Vale do Piranga. De acordo com os autores, esse resultado foi consequência do grande efeito de borda sofrido pelo fragmento. Este resultado aponta que em áreas com perturbações, como é o caso da Fazenda Experimental do Vale do Piranga, há um decréscimo acentuado no estoque de carbono nos indivíduos arbóreos (NELSON et al., 2000; ZHANG et al., 2017). Assim, observa-se que os resultados obtidos estão em consonância com os demais trabalhos nesta linha. Além disso, pode-se inferir que há 
uma alteração significativa no estoque presente quando a área de interesse sofre alguma alteração (SCALON et al., 2012).

Ressalta-se que o fragmento estudado é considerado como uma área de preservação, sendo assim as ações antrópicas são minimizadas. Ademais, áreas com características similares ao fragmento estudado são interessantes na pesquisa para evidenciar fenômenos como a perturbação antrópica sobre a quantidade de estoque de carbono (NELSON et al., 2000). Desta forma, a preservação destes fragmentos é de suma importância no contexto ecológico e prestação de serviços ecossistêmicos como a estocagem de carbono.

A análise diamétrica e populacional do remanescente considerando ambos os anos de mensuração está descrita na tabela 3. Observa-se que o número de indivíduos diminui entre os anos de inventário assim como a quantidade de carbono. Dados os resultados, é importante se atentar a medidas conservacionistas para os ambientes florestais, pois estudos têm comprovado que áreas perturbadas tendem a sequestrar menos carbono em comparação às não perturbadas (MUHATI et al., 2018; PYLES et al., 2018).

TABELA 3 - Análise diamétrica e número de indivíduos por hectare no remanescente de Floresta Estacional Semidecidual Montana em Lavras, Minas Gerais.

$\begin{array}{ccccc}\text { Ano } & \text { DAP }_{\text {Min }} & \text { DAP }_{M}(\mathrm{~cm}) & \text { DAP Máx }_{\text {M }} & \mathrm{N} \\ 2015 & 5 & 13,38 & 78,10 & 882 \mathrm{ind} / \mathrm{ha} \\ 2017 & 5 & 13,37 & 77,30 & 820 \mathrm{ind} / \mathrm{ha}\end{array}$

Sendo: $\mathrm{DAP}_{\mathrm{Min}}=$ Diâmetro mínimo em centímetros; $\mathrm{DAP}_{\mathrm{M}}=$ Diâmetro médio das árvores em centímetros; $\mathrm{DAP}_{\text {Máx }}=$ Diâmetro máximo em centímetros e $\mathrm{N}=$ Número de indivíduos por hectare.

A análise da quantidade de carbono considerando os dois métodos de comparação está explicitada na Tabela 4. Para os dois anos de medição, pode-se observar uma diferença de $1,25 \%$ da quantidade total de carbono para ambos os métodos de tratamento de árvores bifurcadas.

TABELA 4 - Valor de carbono (ton.ha ${ }^{-1}$ ) considerando as metodologias nesse estudo em um remanescente de Floresta Estacional Semidecidual Montana em Lavras, Minas Gerais.

\begin{tabular}{ccc}
\hline Ano & Método & Carbono (ton.ha ${ }^{-1}$ ) \\
\hline \multirow{2}{*}{2015} & 1 & 69,62 \\
& 2 & 68,75 \\
\multirow{2}{*}{2017} & 1 & 68,70 \\
& 2 & 67,83
\end{tabular}

Sendo Método 1 = considerando o cálculo do diâmetro médio quadrático; Método 2 = considerando cada perfilho como um indivíduo.

Em relação ao Incremento de Carbono (IC), houve uma diferença de 1,44\% entre o método 1 e o método 2, explicitado na Tabela 5 . O IC total do monitoramento resultou em $-42,5$ toneladas de carbono, que representa 6,74 ton.ha ${ }^{-1}$ perdidos entre os dois anos de medição. A variação negativa do carbono pode estar atrelada pelos altos índices de mortalidade em relação à baixa taxa de recrutamento no período estudado. 
Observou-se que os indivíduos com diâmetros entre 5 e $6 \mathrm{~cm}$ obtiveram maiores índice de mortalidade. Tais resultados podem estar associados com a competição entre os indivíduos e também a fatores climáticos, como exemplo, o período de estiagem prolongado ocorrente na região, o que influencia diretamente no estabelecimento de indivíduos jovens (GUSTAFSON; STURTEVANT, 2013; RUIZ-BENITO et al., 2013 O'BRIEN et al., 2017; YOUNG et al., 2017).

TABELA 5 - Incremento Periódico Anual de Carbono para os dois anos de medição em um remanescente de Floresta Estacional Semidecidual Montana em Lavras, Minas Gerais.

\begin{tabular}{cc} 
Métodos & Incremento de Carbono 2015-2017 \\
1 & $-2.946,0$ \\
2 & $-2.903,5$ \\
\hline
\end{tabular}

Sendo Método 1= considerando o cálculo do diâmetro médio quadrático; Método 2 = considerando cada perfilho como um indivíduo.

\section{CONCLUSÕES}

Considerando os períodos de mensuração para o fragmento, houve um decréscimo na estocagem de carbono. Contudo, ainda se pode inferir sobre a importância da área como sumidouro de carbono, atuando assim na prestação de serviços ecossistêmicos e mitigação climática. Além disso, por meio deste estudo foram identificadas espécies com potencial na estocagem de carbono e que podem ser introduzidas nos planos de restauração florestal.

Ademais, a estimativa por ambos os procedimentos adotados neste estudo é eficiente, visto que não houveram diferenças significativas. Nesse sentido, a determinação do estoque de carbono pode ser realizada por meio dos indivíduos perfilhados considerando o cálculo do diâmetro geométrico e considerando-os como fustes individuais.

\section{AGRADECIMENTOS}

Ao CNPq pela concessão da bolsa ao primeiro autor, bem como ao Programa de Pós Graduação em Engenharia Florestal e a Universidade Federal de Lavras (UFLA) pela disponibilidade dos dados e estrutura para o desenvolvimento do estudo.

\section{REFERÊNCIAS}

ALVARES, C. A.; STAPE, J. L.; SENTELHAS, P. C.; GONÇALVES, J. L. DE M.; SPAROVEK, G. Köppen's climate classification map for Brazil. Meteorologische Zeitschrift, v. 22, n. 6, p. 711-728, 2013. Disponível em: <https://www.schweizerbart. de/papers/metz/detail/22/82078/Koppen_s_climate_classification_map_for_Brazil>. doi: 10.1127/0941-2948/2013/0507

AMARO, M. A.; SOARES, C. P. B.; SOUZA, A. L. DE; LEITE, H. G.; SILVA, G. F. DA. Estoque volumétrico, de biomassa e de carbono em uma floresta estacional semidecidual em Viçosa, Minas Gerais. Revista Árvore, v. 37, n. 5, p. 849-857, 2013. Disponível em: <https://www.scielo.br/scielo.php?script=sci_arttext\&pid=S0100-6762 2013000500007\&lng=pt\&t|ng=pt> doi: 10.1590/S0100-67622013000500007 
ADAIR, E. C.; HOOPER, D. U.; PAQUETTE, A.; HUNGATE, B.A. Ecosystem context illuminates conflicting roles of plant diversity in carbon storage. Ecology Letters, v. 21, n. 11, p. 1604-1619, 2018. Disponível em: <https://onlinelibrary.wiley.com/doi/abs/ 10.1111/ele.13145>. doi: 10.1111/ele.13145

CARVALHO, D.; ROCHA, A.; GÓMEZ-GESTEIRA, M.; SANTOS, C. S. Offshore wind energy resource simulation forced by different reanalyses: Comparison with observed data in the Iberian Peninsula. Applied Energy, v. 134, p. 57-64, 2014. Disponível em: <https://www.sciencedirect.com/science/article/abs/pii/S0306261914008216>. doi: 10 .1016/j.apenergy.2014.08.018

CHAVE, J.; RÉJOU-MÉCHAIN, M.; BÚRQUEZ, A.; CHIDUMAYO, E.; COLGAN, M. S. et al. Improved allometric models to estimate the aboveground biomass of tropical trees.

Global Change Biology, v. 20, n. 10, p. 3177-3190, 2014. Disponível em: < https://onlinelibrary.wiley.com/doi/abs/10.1111/gcb.12629>. doi: 10.1111/gcb.12629

CORDEIRO, N. G.; PEREIRA, K. M. G.; TERRA, M. DE C. N. S.; MELLO, J. M. DE. Variação temporal do estoque de carbono e volume de madeira em um fragmento de cerrado sensu stricto. Enciclopédia Biosfera, v. 15, n. 28, p. 931-941, 2018. Disponível em: < https://www.conhecer.org.br/enciclop/2018B/AGRAR/Variacao.pdf>. doi: 10.18677/EnciBio_2018B76

CORDEIRO, N. G.; PEREIRA, K. M. G.; PINTO, L. O. R.; TERRA, M. DE C. N. S.; MELLO, J. M. DE. Variação espaço-temporal do estoque de carbono em fragmentos de Cerrado em Minas Gerais. In: JASPER, M. (Ed.). Fontes de Biomassa e Potenciais de uso. Ponta Grossa, Brazil: Atena Editora, 2019. p. 103-116. Disponível em: <https://www.atenaeditora.com.br/post-artigo/21773>. doi: 10.22533/at.ed.29419 160913

CORDEIRO, N. G.; PEREIRA, K. M. G.; TERRA, M. DE C. N. Structural and compositional shifts in Cerrado fragments in up to 11 years monitoring. Acta Scientiarum. Biological Sciences, v. 42, n. 1, p. e48357, 2020. Disponível em: <http://periodicos.uem.br/ojs/index.php/ActaSciBiolSci/article/view/48357>. doi: 10.40 25/actascibiolsci.v42i1.48357

EIBEL, E.; PINHEIRO, R. B. M. Estudo de Caso: Crédito De Carbono. Revista Gestão \& Sustentabilidade Ambiental, v. 4, n. 2, p. 588-601, 2016. Disponível em: < http://www.portaldeperiodicos.unisul.br/index.php/gestao_ambiental/article/view/3226/23 $01>$.

FARIA, I. D. Compensação ambiental: os fundamentos e as normas; a gestão e os conflitos. Brasília: Senado Federal, 2008. 115 p. Disponível em: http://www.terrab rasilis.org.br/ecotecadigital/index.php/estantes/legislacao/1417-compensacao-amb iental-os-fundamentos-e-as-normas-a-gestao-e-os-conflitos.

GROOT, R. DE; FISHER, B.; CHRISTIE, M.; ARONSON, J.; BRAAT, L.; Cet al. Integrating the ecological and economic dimensions in biodiversity and ecosystem service valuation. In: KADEKODI, G. K (Ed.). The Economics of Ecosystems and Biodiversity: Ecological and Economic Foundations. 2010. p. 1-40. Disponível em: 
$<\quad$ http://www.teebweb.org/wp-content/uploads/2013/04/D0-Chapter-1-Integrating-theecological-and-economic-dimensions-in-biodiversity-and-ecosystem-service-valu ation.pdf>

GUSTAFSON, E. J.; STURTEVANT, B. R. Modeling forest mortality caused by drought stress: implications for climate change. Ecosystems, v. 16, n. 1, p. 60-74, 2013. Disponível em: <https://link.springer.com/article/10.1007/s10021-012-9596-1>. doi: 10.1007/s10021-012-9596-1

JOHNSON, J.; ALAHI, A.; FEI-FEI, L. Perceptual Losses for Real-Time Style Transfer and Super-Resolution. European Conference on Computer Vision, v. 9906, p. 694711, 2016. Disponível em: <https://link.springer.com/chapter/10.1007/978-3-319-464756_43>. doi: 10.1007/978-3-319-46475-6_43

KENZO, T.; FURUTANI, R.; HATTORI, D.; TANAKA, S.; SAKURAI, K. et al. Aboveground and belowground biomass in logged-over tropical rain forests under different soil conditions in Borneo. Journal of Forest Research, v. 20, n. 1, p. 197-205, 2015. Disponível em: < https://www.tandfonline.com/doi/abs/10.1007/s10310-014-0465y>. doi: 10.1007/s10310-014-0465-y

KUMAR, L.; MUTANGA, O. Remote sensing of above-ground biomass. Remote Sensing, v. 9, p. 935, 2017. Disponível em: <https://www.mdpi.com/20724292/9/9/935>. doi: 10.3390/rs9090935

LIMA, J. A. DE S.; CARMO, C. A. F. S. DO; KINDEL, A.; MOTTA, P. E. DA. Estimativa de Biomassa e Estoque de Carbono de uma floresta secundária em Minas Gerais. Rio de Janeiro: Embrapa Solos, 2003. Disponível em: <https://www.embrapa.br/solos/busca-de-publicacoes/-/publicacao/338318/estimativ a-de-biomassa-e-estoque-de-carbono-de-uma-floresta-secundaria-em-minas-gerais >

MACDICKEN, K. G.; WOLF, G. V.; BRISCOE, C. B. Standard Research Methods for Multipurpose Trees and Shrubs. Winrock International, 1991. 92 p.

MIRANDA, B. P.; RODERJAN, C. V.; BOTOSSO, P. C.; LONGHI-SANTOS, T.; ANDRADE, V. H. F. Estimativa da biomassa aérea e carbono de llex microdonta em Floresta Atlântica, Paraná, Brasil. BIOFIX Scientific Journal, v. 4, n. 1, p. 58-63, 2019. Disponível em: <https://revistas.ufpr.br/biofix/article/view/63865>. doi: 10.5380/ biofix.v4i1.63865

MIRANDA, D. L. C. DE; MELO, A. C. G. DE; SANQUETTA, C. R. Equações alométricas para estimativa de biomassa e carbono em árvores de reflorestamentos de restauração. Revista Arvore, v. 35, n. 31, p. 679-689, 2011. Disponível em: < https://www.scielo.br/scielo.php?pid=S0100-67622011000400012\&script=sci_arttext >. doi: 10.1590/S0100-67622011000400012

MUHATI, G. L.; OLAGO, D.; OLAKA, L. Quantification of carbon stocks in Mount Marsabit Forest Reserve, a sub-humid montane forest in northern Kenya under anthropogenic disturbance. Global Ecology and Conservation, v. 14, e00383. Disponível em: < https://www.sciencedirect.com/science/article/pii/S23519894173025 00>. doi: 10.1016/j.gecco.2018.e00383 
NAM, V. T.; VAN KUIJK, M.; ANTEN, N. P. R. Allometric equations for abovegroung and belowground biomass estiomations in a Evergreen Forest in Vietnam. Plos One, v. 11, n. 6, p. e0156827, 2016. Diponível em: < https://journals.plos.org/plosone/article ?id=10.1371/journal.pone.0156827>. doi: 10.1371/journal.pone.0156827

NELSON, R. F.; KIMES, D. S.; SALAS, W. A.; ROUTHIER, M. Secondary forest age and tropical forest biomass estimation using thematic mapper imagery: Single-year tropical forest age classes, a surrogate for standing biomass, cannot be reliably identified using single-date tm imagery. BioScience, v. 50, n. 5, p. 419-431, 2000. Disponível em: < https://academic.oup.com/bioscience/article/50/5/419/264202>. doi: 10.1641/0006-3568(2000)050[0419:SFAATF]2.0.CO;2

O'BRIEN, M. J.; ENGELBRECHT, B. M.J.; JOSWING, J.; PEREYRA, G.; SHULDT, B. et al. A synthesis of tree functional traits related to drought-induced mortality in forests across climate zones. Journal of Applied Ecology, v. 54, p. 1669-1689, 2017. Disponível em: < https://besjournals.onlinelibrary.wiley.com/doi/full/10.1111/1365-266 4.12874>. doi: 10.1111/1365-2664.12874

OLIVEIRA, M. DE; RIL, F. L.; PERETTI, C.; CAPELLESSSO, E. S.; SAUSEN, T. L. et al. Biomassa e estoques de carbono em diferentes sistemas florestais no sul do Brasil. Perspectiva, v. 40, n. 149, p. 9-20, 2016. Disponível em: < http://www.uricer.edu.br/site/pdfs/perspectiva/149_542.pdf>.

OLIVEIRA, G. DE C.; FERNANDES-FILHO, E. I. Automated mapping of permanent preservation areas on hilltops. Cerne, v. 22, n. 1, p. 111-120, 2016. Disponível em: < https://www.scielo.br/scielo.php?pid=S0104-77602016000100111\&script=sci_abstra ct >. doi: 10.1590/01047760201622012100

ONU. Adoção do acordo Paris - Convenção Quadro sobre Mudança do Clima. 2015. $42 \mathrm{p}$.

PEDRONI, F.; SANCHEZ, M.; SANTOS, F. A. M. Fenologia da copaíba (Copaifera langsdorffii Desf. - Leguminosae, Caesalpinioideae) em uma floresta semidecídua no sudeste do Brasil. Revista Brasileira de Botânica, v. 25, n. 2, p. 183-194, 2002. Disponível em: < https://www.scielo.br/scielo.php?script=sci_abstract\&pid=S0100$84042002000200007 \&$ Ing=en\&nrm=iso\&tlng=pt $>$. doi: $10.1590 / S 0 \overline{100}-840420020002$ 00007

PYLES, M. V.; PRADO-JUNIOR, J. A.; MAGNAGO, L. F. S.; PAULA, A. DE.; MEIRANETO, J. A. A. Loss of biodiversity and shifts in aboveground biomass drivers in tropical rainforests with different disturbance histories. Biodiversity and Conservation, v. 27, p. 3215-3231, 2018. Disponível em: < https://link.springer.com/a rticle/10.1007\%2Fs10531-018-1598-7\#citeas >. doi: 10.1007/s10531-018-1598-7

R CORE TEAM. R: A language and environment for statistical computing. $\mathbf{R}$ Foundation for Statistical Computing,Vienna, Austria, 2020. Disponível em: $<$ https://www.r-project.org/.>

RÉJOU-MÉCHAIN, M.; TANGUY, A.; PIPONIOT, C.; CHAVE, J.; HÉRAULT, B. Biomass: an $R$ package for estimating above-ground biomass and its uncertainty in 
tropical forests. Methods in Ecology and Evolution, v. 8, p. 1163-1167, 2017. Disponível em: < https://besjournals.onlinelibrary.wiley.com/doi/full/10.1111/2041210X.12753 >. doi: 10.1111/2041-210X.12753

RUIZ-BENITO, P.; LINES, E. R.; GÓMEZ-APARICIO, L.; ZAVALA, M. A.; COOMES, D. A. Patterns and Drivers of Tree Mortality in Iberian Forests: Climatic Effects Are Modified by Competition. PLoS ONE, v. 8, n. 2, p. e56843, 2013. Disponível em: < https://journals. plos.org/plosone/article?id=10.1371/journal.pone.0056843>. doi: 10.1 371/journal.pone.0056843

SANFORD, R. L.; CUEVAS, E. Root growth and rhizosphere interactions in tropical forests. In: Mulkey S.S., Chazdon R.L., Smith A.P. (eds) Tropical Forest Plant Ecophysiology. Springer, Boston, MA. 1996. Disponível em: < https://link.springer.co m/chapter/10.1007/978-1-4613-1163-8_10>.

SCALON, J. D.; OLIVEIRA, C. A. P. DE; MELLO, J. M. DE. Análise espacial de um fragmento florestal baseada no mosaico de Dirichlet. Revista Árvore, v. 36, n. 4, p. 733-740, 2012. Disponível em: < https://www.scielo.br/pdf/rarv/v36n4/a14v36n4.pdf>.

SILVEIRA, E. M. DE O. et al. Estimating Aboveground Biomass Loss from Deforestation in the Savanna and Semi-arid Biomes of Brazil between 2007 and 2017. In: SURATMAN, M. N.; LATIF, Z. A.; OLIVEIRA, G. DE; BRUNSELL, N.; SHIMABUKURO, Y. et al. Forest Degradation Around the World, IntechOpen, 2019 . p. 1-17. Disponível em: < https://www.intechopen.com/books/forest-degradation-around-theworld/estimating-aboveground-biomass-loss-from-deforestation-in-the-sa vanna-and-semi-arid-biomes-of-brazil>. doi: 10.5772/intechopen.85660

SILVEIRA, E. M. DE O.; TERRA, M. DE C. N. S.; TER STEEGE, H.; MAEDA, E. E.; ACERBI JÚNIOR, F. W. et al. Carbon-diversity hotspots and their owners in Brazilian southeastern Savanna, Atlantic Forest and Semi-Arid Woodland domains. Forest Ecology and Management, v. 452, p. 117575, 2019b. Disponível em: <https://www.s ciencedirect.com/science/article/abs/pii/S0378112719309624?via\%3Dihub>. doi: 10 . 1016/j.foreco.2019.117575

SOUZA, M. C. O.; CORAZZA, R. I. Do Protocolo Kyoto ao Acordo de Paris: uma análise das mudanças de regime climático global a partir do estudo da evolução de perfis de emissões de gases de efeito estufa. Desenvolvimento e Meio Ambiente, v. 42, p. 5280. Disponível em: <https://revistas.ufpr.br/made/article/view/51298/34446>. doi: 10.5380/dma.v42i0.51298

SULLIVAN, M. J. P.; TALBOT, J.; LEWIS, S. L.; PHILLIPS, O. L.; QIE, L. et al. Diversity and carbon storage across the tropical forest biome. Scientific Reports, v. 7, n. February, p. 1-12, 2017. Disponível em: <https://www.nature.com/articles/srep39102 >. doi: $10.1038 /$ srep39102

TANAGO, J. G. DE; LAU, A.; BARTHOLOMEUS, H.; HEROLD, M.; AVITABILE, V. et al. Estimation of above-ground biomass of large tropical trees with terrestrial LiDAR. Methods in Ecology and Evolution, v. 9, n. 2, p. 223-234, 2018. Disponível em: < https://besjournals.onlinelibrary.wiley.com/doi/full/10.1111/2041-210X.12904>. doi: 10 
TORRES, C. M. M. E.; JACOVINE, L. A. G.; SOARES, C. P. B., OLIVEIRA NETO, S. N.; SANTOS, R. D. et al. Quantificação de biomassa e estocagem de carbono em uma floresta estacional semidecidual, no parque tecnológico de Viçosa, MG. Revista Árvore, v. 37, n. 4, p. 647-655, 2013. Disponível em: <https://www.scielo.br/scielo.ph p?pid=S0100-67622013000400008\&script=sci_abstract\&tlng=pt>. doi: 10.1590/S010 0-67622013000400008.

TORRES, C. M. M. E.; JACOVINE, L. A. G.; OLIVEIRA NETO, S. N. DE.; SOUZA, A. L. DE; CAMPOS, R. A. et al. Análise fitossociológica e valor de importância em carbono para uma Floresta Estacional Semidecidual. Floresta e Ambiente, v. 24, p. e00099714, 2017. Disponível em: $<$ https://www.scielo.br/scielo.php?script=sci_arttext \&pid=S2179-80872017000100177\&lng=pt\&tIng=pt>. doi: $10.1590 / 2179-8087.099714$

VITAL, M. H. F. Aquecimento global : acordos internacionais, emissões de $\mathrm{CO}_{2}$ e o surgimento dos mercados de carbono no mundo. BNDES Set., Rio de Janeiro, v. 24, n. 48, p. 167-244, 2018. Disponível em: < https://web.bndes.gov.br/bib/jspui/bits tream/1408/16043/2/PRArt214085_Aquecimento\%20global_compl_P.pdf>

YOUNG, D. J. N.; STEVENS, J. T.; EARLES, J. M.; MOORE, J.; ELLIS, A. et al. Longterm climate and competition explain forest mortality patterns under extreme drought. Ecology Letters, v. 20, p. 78-86, 2017. Disponível em: < https://onlinelibrary.wiley.co m/doi/abs/10.1111/ele.12711>. doi: 10.1111/ele.12711

ZANNE, A. E.; LOPEZ-GONZALES, G.; COOMES, D. A.; ILIC, J.; STEVEN, J. et al. Data from: Towards a Worldwide Wood Economics Spectrum. 2009. Disponível em: <https://doi.org/10.5061/dryad.234>. doi: 10.5061/dryad.234

ZELARAYÁN, M. L. C.; CELENTANO, D.; OLIVEIRA, E. C.; TRIANA, S. P.; SODRÉ, D. $\mathrm{N}$. et al. Impacto da degradação sobre o estoque total de carbono de florestas ripárias na Amazônia Oriental, Brasil. Acta Amazonica, v. 45, n. 3, p. 271-282, 2015. Disponível em: < https://www.scielo.br/scielo.php?script=sci_arttext\&pid=S0044-5967 2015000300271\&lng=pt\&tIng=pt>. doi: 10.1590/1809-4392201500432

ZHANG, Y.; CHEN, H. Y. H.; TAYLOR, A. R. Positive species diversity and aboveground biomass relationships are ubiquitous across forest strata despite interference from overstorey trees. Functional Ecology, v. 31, n. 2, p. 419-426, 2017. Disponível em: < https://besjournals.onlinelibrary.wiley.com/doi/full/10.1111/1365-2435.12699 >. doi: $10.1111 / 1365-2435.12699$ 